\title{
Creative Circuits with Deaf Students
}

Brynn Kasper, Emma Koller, Brett Gunderson, AnnMarie Thomas, School of Engineering, University of St. Thomas, St. Paul, MN 55105

\begin{abstract}
$\underline{\text { Abstract }}$
This paper presents an outreach program, designed and delivered by undergraduate engineering students, to introduce Deaf middle and high school students to engineering concepts through a creative circuitry program. Over the course of four weekly two-hour Creative Circuits workshops, students ages 10 to 14 years old worked on projects exploring basic circuitry and programing concepts using Squishy Circuits, Scratch, MaKey MaKey and E-textile learning tools. Instruction was given to the students in American Sign Language with the help of written communication. The team of undergraduate engineering students will present the content of these workshops, reflections by the undergraduate instructing team, and suggestions for running similar programs.
\end{abstract}

\section{$\underline{\text { Introduction }}$}

The Creative Circuits program for Deaf students was developed to introduce a culturally aware STEM program working with Deaf students through American Sign Language to creatively encourage learning about engineering and design. Brynn Kasper, a Mechanical Engineering student minoring in American Sign Language, was the main influence for this program as she has a strong sense of Deaf culture from various classes, Deaf family members and a passion for engineering and design. Metro Deaf School (MDS) in St. Paul, Minnesota was sought out as the partner school because of its proximity to the University of St. Thomas and Kasper's previous experience volunteering there. Collaboration between the Playful Learning Lab and MDS began after Kasper established a connection with the school's after-school program coordinator and proposed the program to administration members.

Research began by searching for existing curricula in engineering designed specifically for Deaf students. Several public forums were found to support the development of STEM vocabulary in American Sign Language ${ }^{1}$. These forums, geared toward teachers and interpreters who work with Deaf students, focus mostly on math and science terms, using user feedback to test the accuracy of the submitted signs. The dictionaries created on these websites were helpful when researching signs for vocabulary to be used in our curriculum, but did not provide a comprehensive collection of engineering terms. Literature on other STEM programs in ASL for Deaf students was limited. The team decided to create a curriculum based on past Playful Learning Lab workshops and adapt programs to be instructed in American Sign Language for Deaf students. 
Two additional undergraduate engineering students, Emma Koller and Brett Gunderson, joined the team once a concrete partnership was made with Metro Deaf School. Koller and Gunderson had little to no previous experience with ASL or Deaf culture. As a collective team, all three undergraduate students had varying levels of experiences with teaching. They worked together with Dr. AnnMarie Thomas to create a curriculum for Creative Circuits.

\section{After-School Program Description}

The Creative Circuits program was designed to provide a STEM program that encouraged interest in engineering and design and was geared specifically toward Deaf students, facilitated in American Sign Language. This objective fit within the existing after-school options that Metro Deaf School provided for its students at a small yearly cost. This Creative Circuits workshop ran in two hour sessions, once a week, for four weeks. The students were not required to attend every class and group attendees varied over the four-week period though the majority of the group regularly attended and were eager to see what each new week would bring. The students that were present ranged from fourth grade to seventh grade. This self-selected group consisted of male students.

\section{Project Goals}

The primary goal of this project was to introduce a culturally aware STEM program working with Deaf students through American Sign Language to encourage learning about engineering and design. As stated by the program title this first run of the program was focused on learning basic circuitry concepts in creative ways. Topics included in the sessions included insulators, conductors, complete and incomplete circuits, parallel and series circuits.

\section{Project Descriptions}

The first project that the students were introduced to was Squishy Circuits ${ }^{2}$. After the instructors had given them a brief introduction to circuitry, including insulators and conductors, the students were then allowed to explore the concepts hands-on by playing with conductive and nonconductive dough and Squishy Circuits kits, including a battery pack, buzzers, LEDs and a motor. 


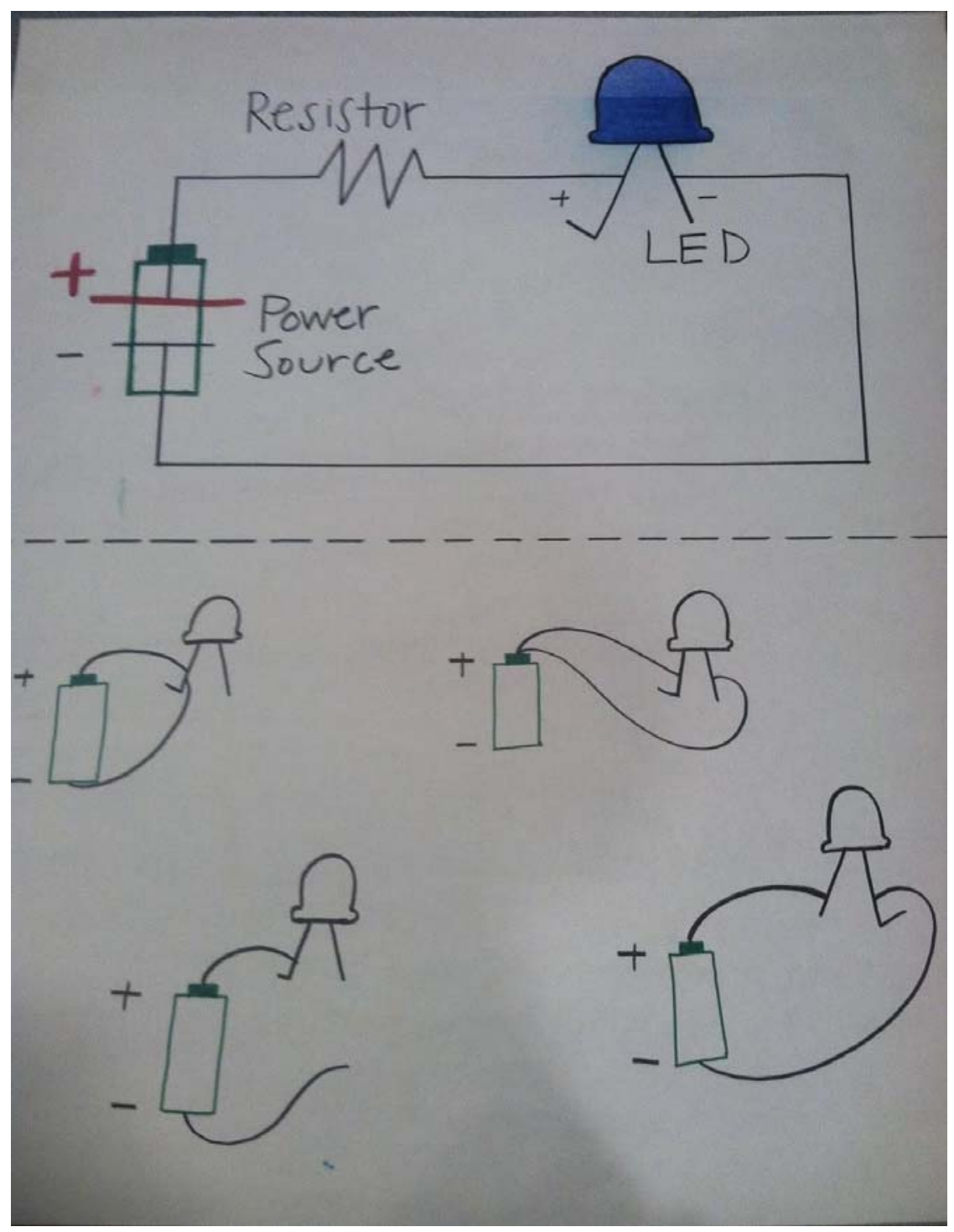

Figure 1

Figure 1 shows one of the posters that the team made to show a complete circuit on top and different ways a circuit could be incomplete below.

Once the group seemed to grasp basic concepts with the help of Squishy Circuits, the team introduced MaKey MaKey ${ }^{3}$, a circuit board geared toward young inventors to replace a keyboard, in conjunction with Scratch, an online programing language for students. The Creative Circuits team had the students use the dough from Squishy Circuits to help them complete the MaKey MaKey circuits.

Hoping to make the final project more memorable, the team used last three sessions to work on E-textiles projects ${ }^{4,5}$. The students would create their own parallel circuit light-up bracelet that they could then take home. To help them create this project the instructors walked through a drawing of what their bracelet could look like. Once the instructors had drawn the bracelet, each 
child was given a piece of paper in the shape of a bracelet, along with a picture of the battery holder and colored circles of paper to represent the LEDs and the button snaps. They were then instructed to glue down the paper parts where they wanted them and then they used crayons to draw the electrical connections of all of the parts to make an imitation circuit. Once their drawings were shown to an instructor, the students were given a kit of the supplies that they would need to complete their bracelets. Each child was given two LEDs, conductive thread, button snaps, a battery holder, and a needle. The students were allowed to choose different colors of felt to use as the base of their bracelet and for decorations that they would add on once their circuit was complete.

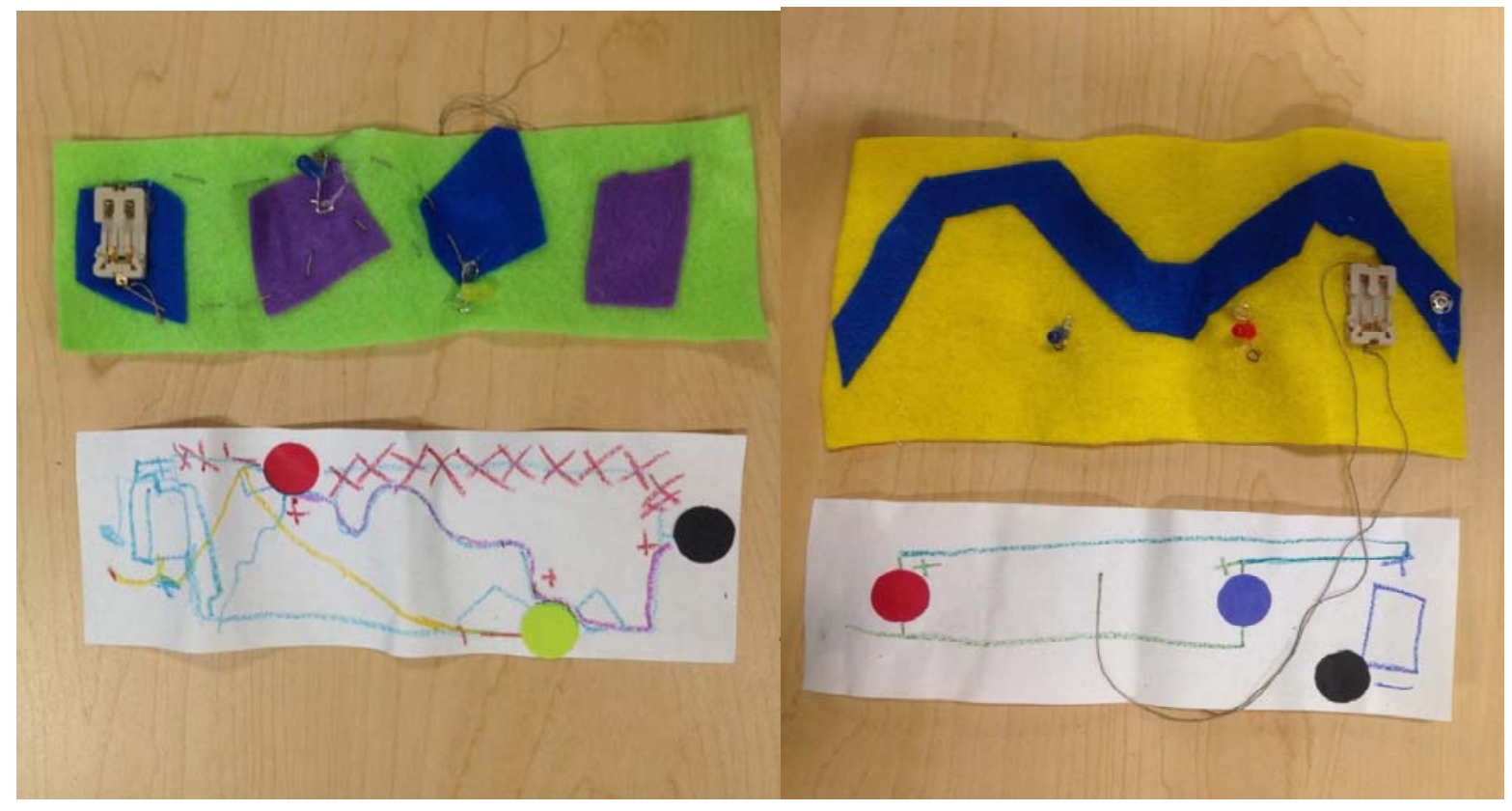

Figure 2

Figure 3

Figures 2 and 3 are examples of two different students' paper bracelet plan paired with the decorated LED bracelet in progress.

\section{$\underline{\text { Reflections and Discussion }}$}

Due to Koller and Gunderson's inexperience with ASL, the instructors had a limited mutual vocabulary to build their curriculum off of. A small number of circuit related words, and the ASL signs for them, were chosen to focus on. These includes "battery”, “circuit”, "LED”, and "electricity". These new English and ASL words were introduced to the students at the same time to create a cohesive understanding of the content for the students while focusing on the student's understanding of the overall concept. Vocabulary words were chosen based on the need of the curriculum, ease of understanding for the students and language capabilities of the language instructor. 
A background in American Sign Language was not required to be a part of the Creative Circuits team, which made most communication between the team and the children difficult. Therefore, support from interpreters and Deaf educators at MDS was welcomed. Kasper created six instructional posters for the students to reference throughout the class. This allowed the Deaf students, who are typically very visual learners, to visually experience the instructions given in addition to the ASL instruction. The posters were helpful when students had questions directed toward a non-fluent signer or non-engineering interpreter or assistant. Their questions could quickly be answered by directing them to one of the visual aids. Since some students were unable to attend every session, the Creative Circuits team was also able to use the posters to quickly review what a student had missed. Kasper would walk through the visuals that had been used in the previous class to remind the students of what they had learned. She then would introduce the new posters she had added for the week. While this worked well for the beginning of the class, once they started to work on a project either the children became too absorbed in the project to look at their surroundings, or there were too many visuals and they had a hard time deciding which would suit their present needs best. The primary goal of the posters was to have something that the children could look at to solve their own questions, not only for the learning, but also to give them the feeling of satisfaction of answering their own question.

The class progression was recorded through the use of review questions at the beginning of each class. The students seemed to understand the circuitry concepts that they were expected to learn as the majority of them actively participated in the review discussions.

Because the sessions had no dedicated learning space, the team found that having too large of a space for its needs made it difficult to keep the group focused as the space was also frequented by other high school students who were not a part of the Creative Circuits group.

The team noted that some children were impatient using Scratch and preferred to just use MaKey MaKey to play games on the computer after their 'keyboard' was set up by a team member. The students then soon realized that MaKey MaKey was inhibiting their ability to play the games and stopped using the learning tool all together after realizing simply using the keyboard was much more efficient.

By having a final project that spans multiple classes, missing a session would leave the student behind in understanding the newly introduced concepts or activity steps. The team did not have the resources to spend copious amounts of time reviewing with students who were behind. Students who completed the activities faster would become restless waiting for the rest of the group to catch up. In the future, a stand-alone project each week or a simple way to catch up students who might miss a session is necessary and would be helpful in keeping all students engaged in the activities. 
Overall the team felt that the first run of the program was a success. Each week the team was told by the after-school program coordinator how the students would question her daily if it was time for "Science Club" again. The team was wary after the first week when they noticed that all of their students were males, realizing that the next project was a bracelet. The team went ahead with their plan to make light-up bracelets and they were overjoyed by the enthusiasm to the project that the children showed. They seemed entranced by the apparent magic that occurred by snapping the bracelet together and having the LED light up. The team feels that because of the joy that they sensed during every class, that the children will look back on this class fondly and remember that science can be fun. They have the best reminder with them, the bracelet. An actual testament to the power of circuitry.

\section{Future Plans and Suggestions}

Due to the high level of interest in the program this year, the team plans to offer the program again. This time including a section for students who attended the first round of classes. This second round will include different and more difficult projects. It has yet to be decided if the future projects will continue to focus just on circuitry as the title "Creative Circuits" implies, or if other areas of science and engineering should be explored in future programs.

The instructors who had no prior ASL experience felt that the program would have run smoother if they were more comfortable with ASL. With that in mind, the team hopes that for the continuation of Creative Circuits, that the original team will continue to create the curriculum. It is hoped that other university students who are studying ASL will be interested in joining the teaching team. This will be piloted in the fall of 2014.

\section{Conclusions}

The outreach program appeared to be successful at meeting the original goal of introducing a culturally aware STEM program working with Deaf students through American Sign Language to encourage learning about engineering and design. The students also seemed to really enjoy our class and the projects that the team had them work on, getting them excited to learn engineering concepts. The team looks forward to growing the program.

\section{Bibliography}

1. ASL-STEM Forum. Deaf and Hard of Hearing Cyber Community project based at the University of Washington. Found at http://aslstem.cs.washington.edu/info/about 
2. Squishy Circuits. University of St. Thomas School of Engineering and the Playful Learning Lab. Web. 1 Aug 2014.

3. MaKey MaKey. JoyLabz, 2012. Web. 1. Aug. 2014.

4. Peppler, Kylie and Diane Glosson. "Stitching Circuits: Learning About Circuitry Through E-textile Materials." Journal of Science Education and Technology 22.5 (2013): 751-763. Web. 1 Aug. 2014.

5. Lovell, Emily. Getting hands-on with soft circuits a workshop facilitator's guide. Found at http://alumni.media.mit.edu/ emme/guide.pdf 